\title{
Représentations \\ et attitudes \\ du public vis-à-vis \\ du cancer
}

> Étant donné le poids du cancer dans la morbidité et la mortalité contemporaines, il apparaît primordial de mesurer les opinions et les perceptions du public ainsi que ses attitudes à l'égard des différents facteurs de risque des maladies cancéreuses. C'est l'objectif de cette revue, à partir des données du Baromètre cancer, enquête menée en 2005 auprès de 4046 personnes représentatives de la population française âgée de 16 ans et plus. Le cancer est la maladie jugée la plus grave par les Français, loin devant le Sida et les maladies cardiovasculaires. Pour les principaux facteurs tels que l'exposition au soleil, l'alcoolisation ou le tabagisme, le risque cancérigène est connu de tous ou presque, mais ce consensus dissimule souvent des croyances solidement ancrées qui relativisent ce risque. Ces croyances peuvent conduire à des comportements dangereux pour la santé à long terme. Par ailleurs, la prolifération des risques perçus peut également susciter ou renforcer une forme de déni du risque. La prégnance de croyances qui relativisent le risque cancérigène invite à prolonger les efforts d'orientation du public vers les pratiques de prévention et à limiter l'isolement et la stigmatisation dont souffrent parfois les personnes atteintes. <

En termes de morbidité et de mortalité, les cancers constituent une préoccupation de santé publique majeure. Ces maladies font ainsi l'objet, depuis 2003, d'un plan national de grande ampleur. L'objectif de cet article est de quantifier, au sein de l'ensemble de la population, les croyances et les opinions à l'égard du cancer ainsi que les attitudes et les comportements face aux facteurs de risque. II s'agit notamment de mettre au jour les jugements de valeur que les individus construisent autour des questions relatives au cancer, dans la mesure où les opinions et les perceptions à l'égard du cancer sont susceptibles de constituer des leviers ou au contraire

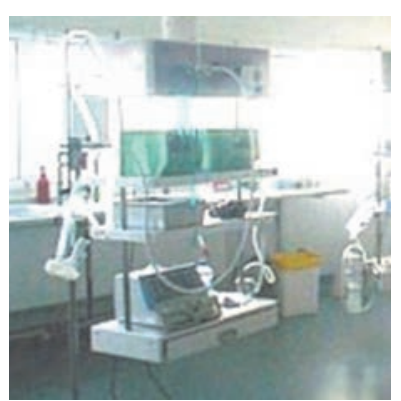

des freins à la prévention ou au repérage précoce de cette pathologie.

\section{Méthode}

Le Baromètre cancer, réalisé par l'Institut national de prévention et d'éducation pour la santé (INPES) en partenariat avec l'Observatoire régional de la santé Pacal, a été mené par téléphone (système $\mathrm{CATI}^{2}$ ) du 26 avril au 15 juin 2005 [1]. II repose sur un échantillon aléatoire de 4046 personnes francophones âgées de 16 ans et plus, représentatif de la population résidant en France métropolitaine.

Les foyers dont le numéro de téléphone est sur liste rouge ont aussi été interrogés (à partir d'une base aléatoire de numéros France Télécom, en ajoutant 1 au dernier chiffre de chaque numéro). Les noms et adresses des personnes correspondant aux numéros de cette liste ont été récupérés dans l'annuaire inversé pour que leur soit envoyée une lettre-annonce, les informant de ce contact et les motivant à participer. Une fois le ménage contacté, l'individu à interroger était sélectionné selon la méthode du prochain anniversaire à venir au sein du foyer. Une société de surveillance était présente tous les jours de l'enquête afin de vérifier la régularité des entretiens.

\footnotetext{
${ }^{1}$ Avec le concours de la Cnamts, la DGS, I'Inca, I'Inserm U357, I'InVS, la Ligue contre le cancer, la Milc et l'OFDT.
}

${ }^{2}$ Computer Assisted Telephone Interview. 
Les données ont été pondérées par le nombre de personnes éligibles dans chaque foyer, et redressées par calage sur marge sur les variables sexe, âge, région et taille d'agglomération, issues du recensement de 1999. Le questionnaire porte sur l'évaluation de la santé perçue, la perception des maladies jugées les plus graves, l'adhésion à la démarche de dépistage, ainsi que sur différents facteurs de risque, en particulier comportementaux. L'exposition au soleil, la consommation d'alcool et de tabac, l'activité physique et la nutrition ont été retenues dans la mesure où ces déterminants ont fait l'objet de campagnes nationales de prévention ces dernières années.

\section{Résultats}

Si la quasi-totalité des Français estime que le cancer est une maladie grave loin devant le Sida et les maladies cardiovasculaires (Figure 1) dont personne n'est à l'abri, seuls quatre sur dix déclarent que c'est une maladie comme les autres et six sur dix estiment qu'elle est souvent héréditaire. Parmi les causes possibles, un quart des enquêtés ne rejette pas la possibilité que certains cancers soient contagieux (Figure 2). Juger que le cancer est une maladie comme les autres ne dépend ni du sexe ni du diplôme, mais cette opinion est plus répandue chez les plus personnes plus âgées.

Relativement aux données épidémiologiques actuelles, le risque perçu de développer un cancer au cours de sa vie apparaît assez proche chez les femmes ( $35 \%$ selon les déclarations versus $37 \%$ selon les données épidémiologiques) mais inférieur chez les hommes (respectivement $39 \%$ versus $47 \%$ ). Ce risque perçu varie peu selon les caractéristiques sociodémographiques. II est en revanche plus élevé parmi les enquêtés qui ont déjà eu un proche atteint et varie aussi selon le mode de vie: les individus qui ont des conduites considérées «à risque » perçoivent un risque plus élevé, à l'inverse de ceux qui adoptent des comportements considérés comme préventifs.

\section{Tabagisme et exposition au soleil}

S'agissant des facteurs susceptibles de favoriser la survenue d'un cancer, la quasi-totalité des enquêtés identifie le tabagisme et l'exposition au soleil sans protection (Figure 3). Si le statut tabagique n'a pas d'incidence sur cette connaissance, seuls $14,4 \%$ des fumeurs actuels pensent que le risque de cancer dû à la cigarette existe dès que l'on fume une cigarette par jour, tandis qu'un sur quatre situe ce seuil à au moins vingt cigarettes par jour.

En outre, un enquêté sur trois craint d'avoir un jour un cancer dû au tabagisme, crainte partagée, à cause du tabagisme passif, par un quart des non-fumeurs. Inversement, trois fumeurs sur dix ne redouteraient pas d'avoir un jour un cancer dû au tabac, le plus souvent parce qu'ils pensent fumer trop peu.

Bien que l'exposition au soleil soit le deuxième facteur de risque le plus souvent cité, les connaissances des Français en la matière restent perfectibles: seuls quatre sur dix savent qu'il faut se méfier du soleil entre 12 et 16 heures, et si $88 \%$ savent que les rayonnements solaires sont particulièrement forts en juillet, et $80 \%$ pour le mois d'août, cette proportion tombe à $40 \%$ pour juin et $6 \%$ pour mai, mois également à risque. Les hommes, les personnes d'âges intermédiaires et les plus diplômées sont mieux informés à ce sujet.

Le recours systématique aux différents moyens de protection envisagés dans le questionnaire reste minoritaire: pour se protéger lors d'une journée d'été ensoleillée, $42 \%$ des enquêtés déclarent porter systématiquement des lunettes de soleil ; $31 \%$ disent éviter de s'exposer durant les heures les plus ensoleillées ; $21 \%$ restent à l'ombre d'un parasol ; $23 \%$ gardent un chapeau ou une casquette; enfin, seuls $14 \%$ mettraient de la crème solaire toutes les heures. Au total, $63 \%$ disent utiliser de façon systématique au moins l'un de ces moyens de protection.

Toutefois, un quart des Français pense que s'exposer aux UV en cabine avant les vacances protège des coups de soleil. Cette croyance est plus fréquente aux âges intermédiaires (qui sont aussi les âges durant lesquels cette pratique est la plus courante). En outre, huit enquêtés sur dix savent que le soleil peut provoquer un vieillissement prématuré de la peau, mais un tiers pense que les coups de soleil de l'enfance bien soignés sont sans conséquence à l'âge adulte, et un quart estime que les coups de soleil préparent la peau en la rendant moins vulnérable au soleil. Les femmes, les personnes âgées de 35 à 54 ans et les personnes

Figure 1. Maladies citées comme faisant partie des trois plus graves (en pourcentages). 


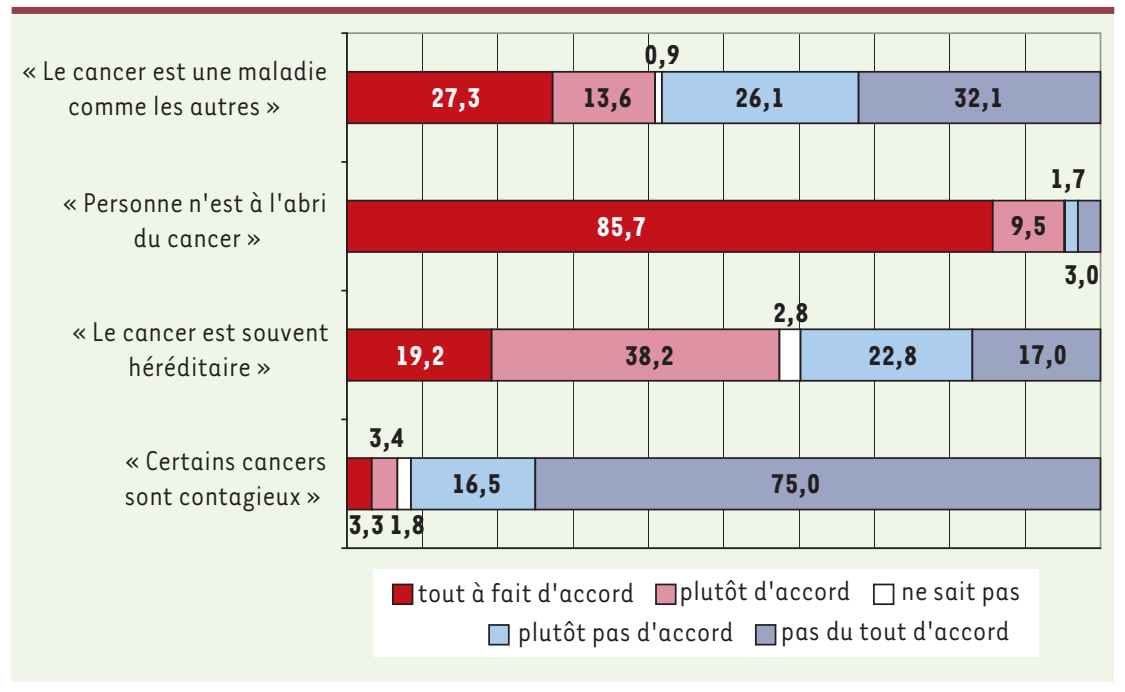

Figure 2. Quelques opinions générales sur le cancer (en pourcentages).

la santé que de consommer des boissons alcoolisées, et plus de la moitié estime que la pollution provoque plus de cancers que l'alcool. De même, pour un quart d'entre eux, consommer des boissons alcoolisées est mauvais pour la santé seulement en cas d'ivresse; tandis que $85 \%$ jugent que les accidents de la route et la violence sont les principaux risques liés à l'alcool. Enfin, pour $59 \%$, boire un peu de vin est meilleur pour la santé que ne pas en boire du tout et pour

plus diplômées sont à la fois plus enclines à juger que le soleil fait vieillir prématurément la peau et moins enclines à sous-estimer les conséquences des coups de soleil de l'enfance ou à prêter des vertus protectrices aux coups de soleil. Ces croyances sont cohérentes avec le risque perçu : ceux qui jugent que les coups de soleil de l'enfance bien soignés sont sans conséquence, ou que les coups de soleil rendent moins vulnérable au soleil estiment moins souvent que l'exposition non protégée au soleil est un facteur de risque de cancer.

\section{Facteurs environnementaux}

Parmi les causes les plus souvent attribuées au cancer viennent ensuite des facteurs environnementaux: la pollution de l'air, les aliments traités avec des produits chimiques (pour environ neuf enquêtés sur dix), la proximité d'une centrale nucléaire (72\%), ainsi qu'un facteur comportemental (boire plus de trois verres d'une boisson alcoolisée par jour : $81 \%$ ) (Figure 3). Au total, $86 \%$ des enquêtés se disent bien informés sur les effets de l'alcool sur la santé. Cette perception du risque décline avec l'âge, elle est aussi plus rare parmi les moins diplômés et les plus gros consommateurs d'alcool. On constate toutefois une tendance à minimiser les risques associés à l'alcool, dans la mesure où pour $56 \%$ des enquêtés, boire des boissons alcoolisées ne provoque le cancer que si l'on boit beaucoup et sur la durée. En outre, la moitié des Français souligne que certaines personnes peuvent consommer beaucoup d'alcool toute leur vie sans jamais tomber malade. Au total, les trois quarts des enquêtés se disent d'accord avec au moins l'une de ces deux opinions qui minimisent les risques associés à l'alcool.

Ces risques sont aussi fréquemment relativisés: plus des deux tiers des Français jugent que boire des sodas ou manger des hamburgers est aussi mauvais pour
$51 \%$ ce sont surtout les alcools forts qui sont mauvais pour la santé. Ces opinions qui renvoient à une relativisation des effets de l'alcool sur la santé sont associées à une moindre perception des effets cancérigènes de l'usage d'alcool. Elles sont davantage défendues par les buveurs quotidiens.

Parmi les caractéristiques sociodémographiques liées aux représentations et aux attitudes face au cancer, le niveau de diplôme, et dans une moindre mesure l'âge, ressortent assez fréquemment. Les personnes plus diplômées d'âge intermédiaire semblent ainsi mieux informées et plus enclines à adopter des comportements de prévention.

\section{Discussion}

On constate que les Français craignent particulièrement le cancer, ce qui avait déjà été observé [2-6]. On sait aujourd'hui que l'hérédité, l'environnement et surtout le style de vie jouent un rôle dans son étiologie [7, 8]. La compréhension des facteurs comportementaux est donc cruciale pour concevoir des actions préventives qui tiennent compte des croyances et des perceptions du public [9, 10]. Ces actions doivent s'intégrer dans les schèmes d'appréhension profanes, prismes au travers desquels les messages préventifs sont interprétés [11]. Notre enquête montre aussi la persistance de représentations péjoratives liées au cancer (qui peut avoir des effets délétères pour les personnes atteintes [12]), ainsi que la croyance en de multiples agents cancérigènes. $0 r$, certaines de ces opinions peuvent freiner ou favoriser les comportements jugés favorables à la santé. Par exemple, la croyance en la contagiosité du cancer pourrait diminuer le risque perçu pour soi et favoriser les conduites à risque (car il suffirait alors d'éviter les personnes atteintes pour éviter la maladie) (Figure 3).

Pour l'exposition au soleil, l'alcoolisation et le tabagisme, le risque cancérigène est reconnu par une très large majorité des enquêtés, qui se dit bien informée, mais ce consensus dissimule souvent des idées reçues solidement ancrées, qui peuvent soutenir des conduites à risque. Nos résultats suggèrent aussi que la prolifération des risques perçus peut induire une relativisation des dangers de certaines de ces conduites [13] : ainsi, les fumeurs et les buveurs quotidiens 
sont plus enclins à juger cancérigènes des facteurs environnementaux et/ou psychologiques. Certaines croyances propices au déni du risque tabagique s'avèrent largement répandues [14], même parmi les nonfumeurs. Les deux tiers des Français pensent ainsi que respirer l'air des villes est aussi mauvais pour la santé que de fumer des cigarettes, que I'on peut fumer toute sa vie sans jamais être malade, ou qu'un fumeur peut éviter les effets nocifs du tabac s'il s'arrête à temps. Ces croyances sont souvent fondées sur l'observation de leur propre comportement, ce qui les rend sans doute plus difficiles à remettre en cause. Les fumeurs disposent en effet de tout un arsenal discursif pour relativiser ce risque pour eux-mêmes, tout en l'acceptant pour les autres fumeurs [15-17]. De futures campagnes d'information pourraient cibler certaines de ces croyances qui relativisent le risque, notamment celles liées aux seuils de dangerosité du tabagisme.

Si le tabagisme a reculé en France, grâce notamment aux fortes hausses du prix des cigarettes de 2003 et 2004, beaucoup d'efforts restent à faire dans le domaine préventif. En particulier, les médecins pourraient systématiquement engager une discussion sur le tabac avec leurs patients fumeurs. À l'instar du tabagisme, la consommation d'alcool recule depuis plusieurs décennies, à la fois grâce à des mutations du mode de vie et de l'alimentation, mais aussi grâce aux campagnes de prévention, en dépit des efforts des acteurs économiques du domaine en direction des plus jeunes avec de nouveaux produits tels que les prémix. Si les enquêtés estiment pour la plupart que l'alcool peut avoir des effets cancérigènes, ils sont aussi très nombreux, notamment à l'adolescence [18, 19], à partager des opinions susceptibles de nourrir un déni du risque. Ces opinions sont sans doute d'autant plus convaincantes qu'elles ne sont pas «fausses» et sont relayées dans les médias et parfois même, indirectement, par les campagnes de prévention (par exemple lorsque l'accent est mis, avec raison, sur les accidents et la violence engendrés par l'abus d'alcool).

S'agissant enfin du risque cancérigène associé au soleil, il est connu par tous ou presque, mais des croyances erronées persistent, comme l'idée que l'exposition au soleil des enfants est «bonne pour la santé »[20]. Les usages sociaux du soleil et les conceptions du corps qui s'y rapportent constituent des obstacles redoutables pour la prévention, comme l'a montré une abondante littérature [21-25]. Ils pourraient à ce titre être ciblés par la prévention. II est aussi envisageable de cibler les personnes selon leur sexe et leur âge, pour promouvoir des moyens de protection adaptés à leurs préférences vestimentaires comme aux activités au cours desquelles elles s'exposent au soleil.

L'ensemble de ces résultats conduit à s'interroger sur la construction des messages de prévention, dans un contexte marqué par la multiplication des risques et des facteurs de risque. Par exemple, lorsque I'on informe simultanément le public sur plusieurs facteurs associés à une même maladie (comme l'alcool et la pollution pour le cancer), ou sur plusieurs risques associés à une même conduite (violence, accidents, cancer pour l'alcool), le risque pourrait être de susciter des effets d'éviction entre messages, contribuant à brouiller ou à conduire à relativiser certains messages préventifs.

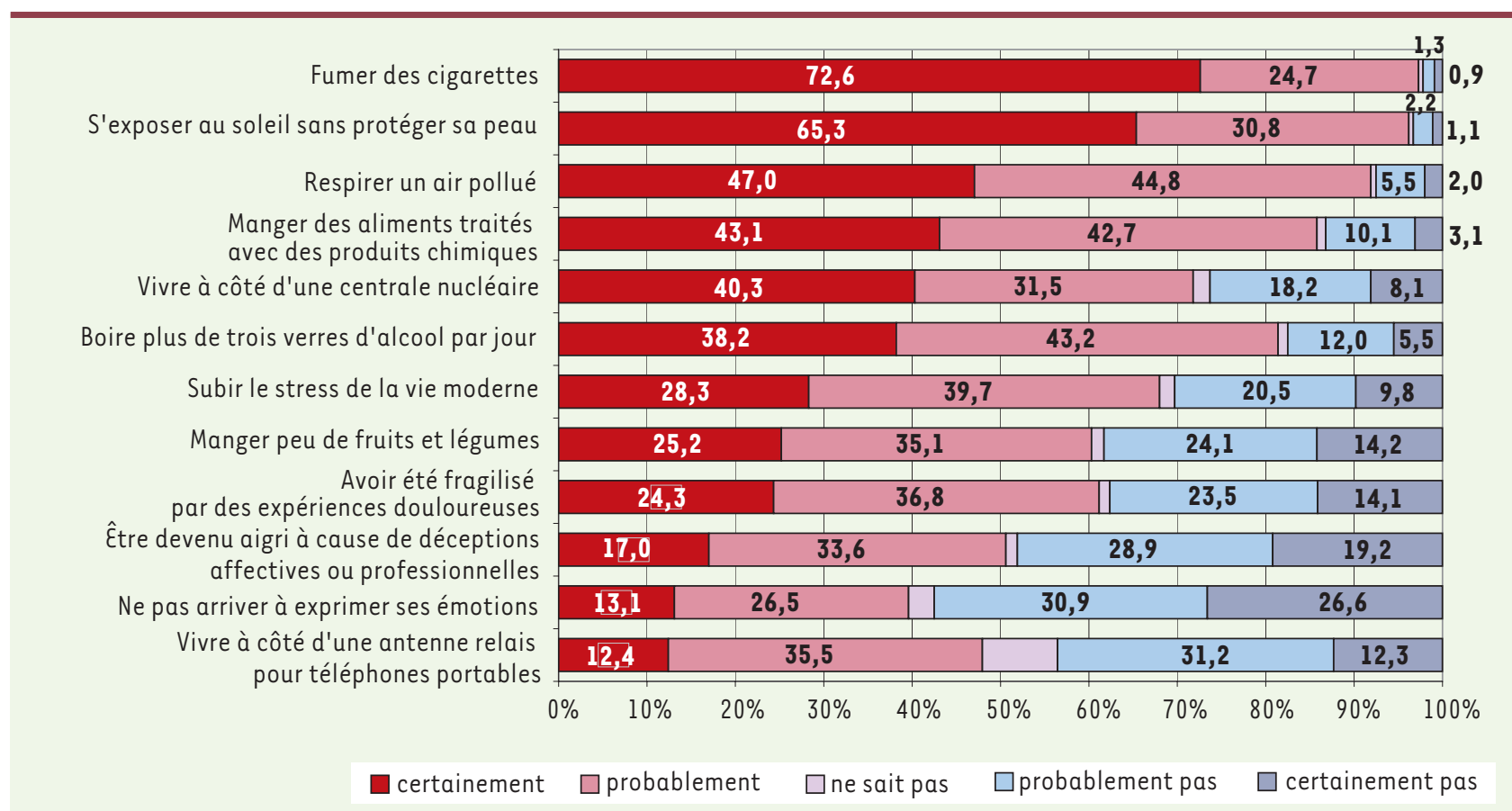

Figure 3. Opinions sur les causes possibles du cancer (en pourcentages). 


\section{Conclusion}

Les représentations du public ont un rôle pratique : elles constituent d'une part un cadre cognitif cohérent qui donne du sens à l'information reçue et à l'expérience vécue, et d'autre part un guide pour l'action [26]. La résistance de certaines idées invite à prolonger les efforts d'information sur ces maladies afin de limiter l'isolement et la stigmatisation dont souffrent parfois les personnes atteintes. Le déni du risque ne reflète pas forcément un manque d'information, mais s'apparente aussi à une construction cognitive qui donne de la cohérence aux conduites: par exemple, la prévention ne doit pas sous-estimer l'aptitude des fumeurs à maintenir leur déni du risque tabagique.

À l'avenir, les efforts préventifs pourraient cibler certaines perceptions, ou au moins prendre en compte le fait que l'information a parfois des effets inattendus, voire indésirables (en particulier si elle nourrit une prolifération des facteurs perçus comme cancérigènes, qui en retour incite à relativiser certaines conduites individuelles à risque). Cela donnerait un cadre pédagogique aux acteurs de prévention qui ont alors la possibilité d'être plus attentifs aux convictions du public auquel s'adressent les interventions préventives. $\diamond$

\section{SUMMARY}

Representations and attitudes toward cancer in the French general population

Cancer has become a major public health issue. It is thus crucial to measure the general population's behaviours, opinions and perceptions about cancer and its associated risk factors. This article describes some of the main findings of a 2005 French survey $(n=4,046)$. Cancer is considered by a large majority to be the most serious disease, far before HIV/AIDS and cardiovascular diseases. The carcinogenic risk that is associated to main risk factors, such as sun exposure, tobaccosmoking and alcohol use appears to be well-known. However, many people justify dangerous behaviours with strongly-anchored beliefs, which maintain dangerous behaviours for health on the long-term. What's more, the perception of risk proliferation can also generate risk denial. Because self-exempting beliefs are still widespread within the general opinion, it is essential to continue public health information campaigns dedicated to cancer prevention, so as to induce better prevention practices within the general population and to reduce stigmatisation and isolation experienced by cancer patients. If risk denial is not systematically a consequence of a lack of information, it is generally associated to a cognitive construction that gives coherence to behaviours. $\diamond$

\section{RÉFÉRENCES}

1. Guilbert P, Peretti-Watel P, Beck F, Gautier A. Baromètre cancer 2005. Saint-Denis : INPES, 2006.

2. Pinell P. Naissance d'un fléau, histoire de la lutte contre le cancer en France, 1890-1940. Paris: Métailié, 1992.

3. Herzlich C. Santé et maladie. Analyse d'une représentation sociale. Paris: Mouton, 1969.

4. Pollak M, Dab W, Moatti JP. Systèmes de réaction au Sida et action préventive. Sciences Sociales et Santé $1989 ; 7: 111-40$.

5. Di Mola G, Crisci MT. Attitudes towards death and dying in a representative sample of the Italian population. Palliative Med $2001 ; 15$ : 372-8.

6. Le Brun J. Cancer serpit. Recherches sur la représentation du cancer dans les biographies spirituelles féminines du XVIIe siècle. Sciences Sociales et Santé $1984 ; 2$ : 9-31.

7. Serraino D. Vieillissement et cancer : association ou causalité ? Med Sci (Paris) 2007 ; $23: 26-8$.

8. Stoppa-Lyonnet $D$, Lenoir $G$. Prédispositions génétiques aux cancers : actualités et perspectives en 2005. Med Sci (Paris) $2005 ; 21: 962-8$.

9. Nkondjock A, Ghadirian P. Facteurs de risque du cancer du sein. Med Sci (Paris) 2005 : $21: 175-80$.

10. Weiman J, Peitrie KJ, Moss-Morris N, Horne R. The illness perception questionnaire: a new method for assessing the cognitive representation of illness. Psychology Health 1996; $11: 431-45$.

11. Moatti JP, Dab W, Pollak M, et al. Les attitudes et comportements des français face au SIDA. La Recherche $1990 ; 223: 888-95$.

12. Chapple A, Ziebland S, McPherson A. Stigma, shame, and blame experienced by patients with lung cancer: qualitative study. Br Med J $2004 ; 328: 1470$.

13. Peretti-Watel P. Sociologie du risque. Paris: Armand Colin, 2000.

14. Peretti-Watel P, Constance J, Guilbert P, et al. Smoking too few cigarettes to be at risk? Smokers' perceptions of risk and risk denial, a French survey. Tobacco Control 2007 ; $16: 351-6$.

15. Sutton SR. How accurate are smokers' perceptions of risk? Health Risk Society 1999 ; $1: 223-30$.

16. Weinstein ND, Marcus SE, Moser RP. Smokers' unrealistic optimism about their risk. Tobacco Control $2005 ; 14: 55-9$.

17. Oakes W, Chapman S, Borland R, Balmford J, Trotter L. «Bulletproof skeptics in life's jungle »: which self-exempting beliefs about smoking most predict lack of progression towards quitting? Prev Med $2004 ; 39: 776-82$.

18. Parker H, Alridge J, Measham F. Illegal Leisure: the normalization of adolescent recreational drug use. London: Routledge, 1998.

19. Benthin A, Slovic P, Moran P, et al. Adolescent health-threatening and health-enhancing behaviours: a study of word association and imagery. J Adolescent Health $1995 ; 17$ : 143-52.

20. Grob JJ, Guglielmina C, Gouvernet J, et al. Study of sunbathing habits in children and adolescents: application to the prevention of melanoma. Dermatology $1993 ; 186: 94-8$.

21. Aquilina S, Gauci AA, Ellul M, Scerri L. Sun awareness in Maltese secondary school students. J Eur Acad Dermatol $2004 ; 18: 670-5$.

22. Branstrom R, Ullen $H$, Brandberg $Y$. Attitudes, subjective norms and perception of behavioural control as predictors of sun-related behaviour in Swedish adults. Prev Med 2004 ; 39 : 992-9.

23. Melia J, Bulman A. Sunburn and tanning in a British population.J Public Health Med 1995 ; $17: 223-9$.

24. Wichstrom L. Predictors of Norwegian adolescents' sunbathing and use of sunscreen. Health Psychol $1994 ; 13: 412-20$.

25. Owen T, Fitzpatrick D, Dolan 0, Gavin A. Knowledge, attitudes and behaviour in the sun: the barriers to behavioural change in Northern Ireland. Ulster Medical Journal 2004 ; 73: 96-104.

26. Jodelet D. Les représentations sociales. Paris: Presses universitaires de France, 1989.

\section{TIRÉS À PART}

F. Beck 